\title{
Tunable optical gradient trap by radial varying polarization Bessel-Gauss beam
}

\author{
Xiu-Min Gao ${ }^{1,2}$, Song $\mathrm{Hu}^{1}$, Jin-Song $\mathrm{Li}^{3}$, Zuo-Hong Ding ${ }^{2}$, Han-Ming Guo ${ }^{2}$, Song-Lin Zhuang ${ }^{2}$ \\ ${ }^{1}$ Electronics \& Information College, Hangzhou Dianzi University, Hangzhou, China; \\ ${ }^{2}$ Optics \& Electronics College, University of shanghai for Science and Technology, Shanghai, China; \\ ${ }^{3}$ Optics \& Electronics College, China Jiliang University, Hangzhou, China. \\ Email: xiumin_gao@yahoo.com.cn
}

Received 28 December 2009; revised 10 January 2010; accepted 12 January 2010.

\begin{abstract}
Optical tweezers play an important role in many domains, especially in life science. And optical gradient force is necessary for constructing optical tweezers. In this paper, the optical gradient force in the focal region of radial varying polarization BesselGauss beam is investigated numerically by means of vector diffraction theory. Results show that the beam parameter and vary rate parameter that indicates the change speed of polarization rotation angle affect the optical gradient force pattern very considerably, and some novel force distributions may come into being, such as multiple force minimums, force ring, and force crust. Therefore, the focusing of radial varying polarization Bessel-Gauss beam can be used to construct optical traps.
\end{abstract}

Keywords: Optical Gradient Force; Bessel-Gauss Beam; Radial Varying Polarization; Vector Diffraction Theory

\section{INTRODUCTION}

Optical tweezers technique has accelerated many major advances in numerous areas of science, especially in life science, since Ashkin developed optical tweezers system 1980s $[1,2,3,4]$. Optical tweezers can offer a very convenient, noninvasive, and non-contact access to processes at the microscopic scale [5], and a number of approaches have been proposed to constructing optical trap, such as generalized phase-contrast technique and holographic optical tweezers arrays [6,7]. In optical trapping system, it is usually deemed that the forces exerted on the particles in light field include two kinds of forces, one is the gradient force, which is proportional to the intensity gradient; the other is the scattering force, which is proportional to the optical intensity [8]. Therefore, optical gradient force is necessary for constructing optical tweezers, and the tunable focal intensity distribution predicts that the position of optical trap may be controllable.
It is well known that Bessel beams provide valid solutions to Helmholtz equation, and have attracted a lot of attention $[9,10,11]$ for their non-diffracting property. And these beams are easily generated external to the laser cavity by illuminating an axicon with a Gaussian beam [12]. In this paper, the optical gradient force in the focal region of radial varying polarization Bessel-Gauss beam is investigated numerically by means of vector diffraction theory. The principle of the focusing this non-spiral vortex Gaussian beam is given in Section 2. Section 3 shows the simulation results and discussions. The conclusions are summarized in Section 4.

\section{PRINCIPLE OF FOCUSING RADIAL VARYING POLARIZATION BESSEL-GAUSS BEAM PRINCIPLE OF THE FOCUSING GAUSSIAN}

According the vector diffraction theory [13,14], the electric field in focal region of the radial varying polarization Bessel-Gauss beam can be written in the form as,

$$
\vec{E}(r, \phi, z)=E_{r} \vec{e}_{r}+E_{z} \vec{e}_{z}+E_{\varphi} \vec{e}_{\varphi}
$$

where $\vec{e}_{r}, \vec{e}_{z}$, and $\vec{e}_{\varphi}$ are the unit vectors in the radial, azimuthal, and propagating directions, respectively. $E_{r}$, $E_{z}$, and $E_{\varphi}$ are amplitudes of the three orthogonal components and can be expressed as

$$
\begin{gathered}
E_{r}(r, z)=A \cos \phi \int_{0}^{\alpha} \cos ^{1 / 2} \theta P(\theta) \sin (2 \theta) \\
\cdot J_{1}(k r \sin \theta) \exp (i k z \cos \theta) d \theta \\
E_{z}(r, z)=2 i A \cos \phi \int_{0}^{\alpha} \cos ^{1 / 2} \theta P(\theta) \sin ^{2}(\theta) \\
\cdot J_{0}(k r \sin \theta) \exp (i k z \cos \theta) d \theta \\
E_{\varphi}(r, z)=2 A \sin \phi \int_{0}^{\alpha} \cos ^{1 / 2} \theta P(\theta) \sin (\theta) \\
\cdot J_{1}(k r \sin \theta) \exp (i k z \cos \theta) d \theta
\end{gathered}
$$


where $r$ and $z$ are the radial and $z$ coordinates of observation point in focal region, respectively. $k$ is wave number. Here $P(\theta)$ is the pupil apodization function [15],

$$
P(\theta)=J_{1}\left(\frac{2 \beta_{1} \sin \theta}{N A}\right) \cdot \exp \left[-\left(\frac{\beta_{2} \sin \theta}{N A}\right)^{2}\right]
$$

$\alpha=\arcsin (N A)$, which practically indicates the radius corresponding to each section zone of the cylindrical vector beam. $\phi$ is the polarization rotation angle from radial direction. As the function of convergence angle $\theta$, and $\phi$ is in the form of,

$$
\phi=C \cdot \frac{\sin (\theta)}{\sin (\alpha)} \cdot \pi
$$

where $C$ is viable rate parameter that indicates the change speed of polarization rotation angle. Based on the optical intensity distribution in focal region, the gradient force trap can be expressed as $[1,16]$,

$$
F_{\text {grad }}=\frac{n_{b}^{2} r^{3}}{2} \cdot\left(\frac{m^{2}-1}{m^{2}+2}\right) \nabla|\vec{E}(r, \varphi, z)|^{2}
$$

where $r$ is the radius of trapped particles, $n_{b}$ is the refraction index of the surrounding medium, and $m$, the relative index of refraction, equals to the ratio of the refraction index of the particle $n_{p}$ to the refraction index of the surrounding medium $n_{b}$. Gradient force points in the direction of the light intensity gradient when the diffractive index of particles is bigger than that of surrounding medium, i.e. $n_{p}>n_{b}$. Therefore, the gradient force pattern can be computed numerically by substituting Eq. 1 into Eq. 6.

\section{RESULTS AND DISCUSSIONS}

Without losing generality and validity, it is supposed that $A=1, N A=0.95$, and $\beta_{2}=1$. It should be noted that in this paper $V r$ and $V z$ denote radial and axial coordinates, and the distance is $k^{-1}$, where $k$ is wave number of the incident BG beam. The intensity distribution and corresponding optical gradient force pattern for $\beta_{1}=2.0$ and $C=1.0$ are firstly calculated and illustrated in Figure 1. Arrows in this figure indicate the force direction under condition of the diffractive index of particles is bigger than that of surrounding medium. It can be seen that the intensity distribution turns on the focal ring, as shown in Figure 1(a), which can be used to construct ring-shape focal trap, given in Figure 1(b).

Now the effect of the parameter $\beta_{1}$ on optical gradient is investigated. It is chosen that $\beta_{1}=2.5$ in the following calculation. The corresponding intensity distribu-

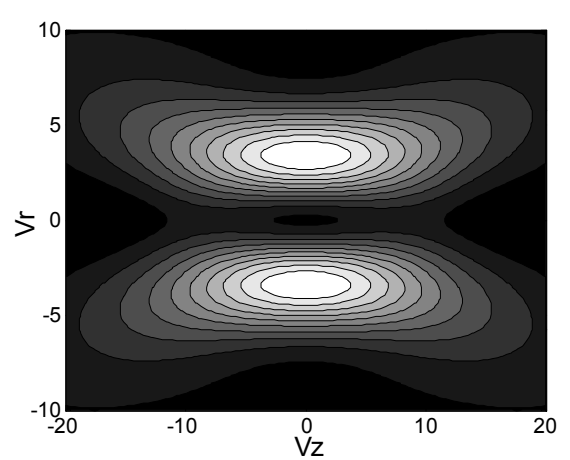

(a)

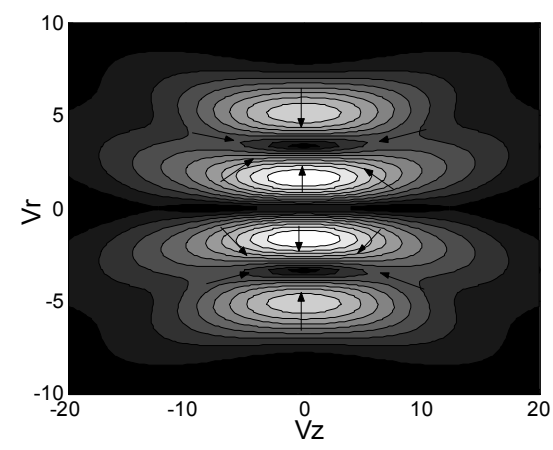

(b)

Figure 1. The (a) intensity distribution and corresponding (b) optical gradient force pattern for $\beta_{1}=2.0$ and $C=1.0$, respectively.

Arrows indicate the force direction.

tion and gradient force pattern are given in Figure 2. We can see that the focal ring extends along optical axis, and there three weak on-axis peaks. From Figure 2(b), it can be seen that there is one cylindrical crust trap, and multiple weak traps on axis. Therefore, parameter $\beta_{1}$ affects the Bessel-Gauss beam, in turn can alter the optical gradient force pattern considerably.

In order to get insight into the optical gradient force in the focal region of radial varying polarization Bessel-Gauss beam more deeply, different $C$ is also considered in calculation. From Figure 3(a), it can be seen that one optical intensity crust comes into being under condition of $C=0.2$, namely, one local intensity minimum occurs. Figure 3(b) illustrates the corresponding optical gradient force pattern that is in practice force crust pattern. So, the parameter $C$ can be used to alter optical gradient force pattern in focal region of the BesselGauss beam.

In our theoretical investigation, more values of $\beta_{1}$ and $C$ are studied. And many novel optical gradient force patterns can occur. This paper only gives several typical cases. Figure 4 illustrates the intensity distribution and corresponding optical gradient force patternfor $\beta_{1}=3.5$ and $C=1.0$. One intensity distorted cylinder 


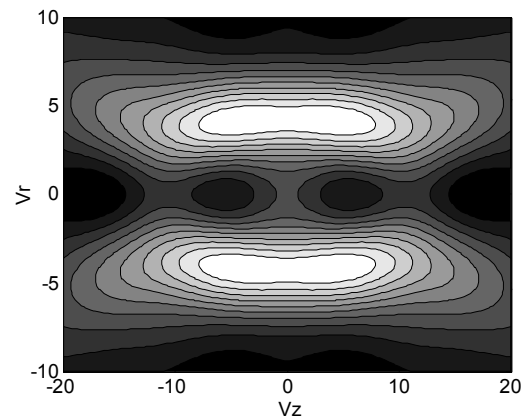

(a)

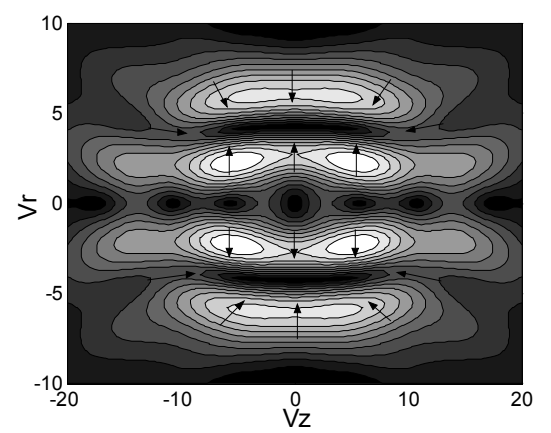

(b)

Figure 2. The (a) intensity distribution and corresponding (b) optical gradient force pattern for $\beta_{1}=2.5$ and $C=1.0$, respectively. Arrows indicate the force direction.

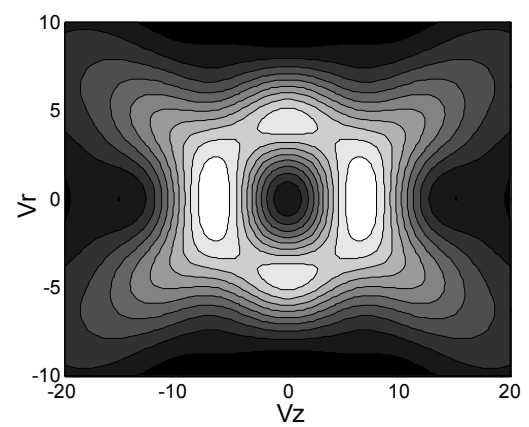

(a)

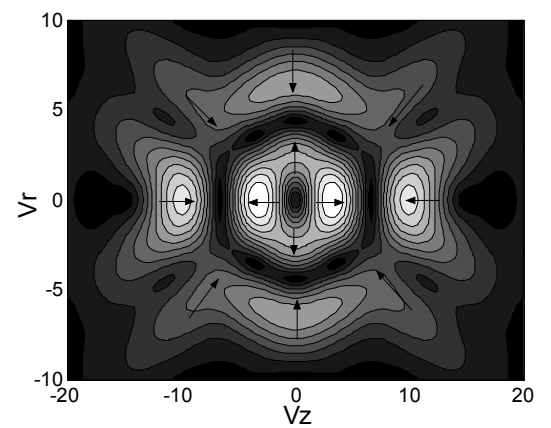

(b)

Figure 3. The (a) intensity distribution and corresponding (b) optical gradient force pattern for $\beta_{1}=2.5$ and $C=0.2$, respectively. Arrows indicate the force direction.

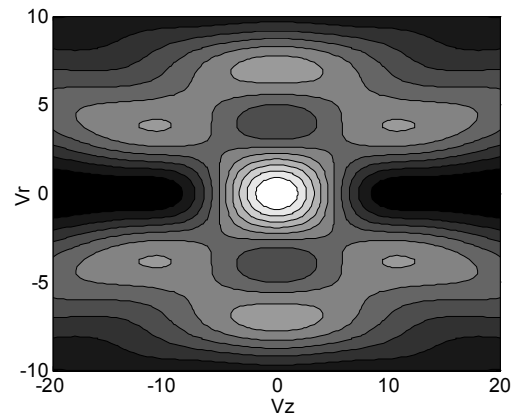

(a)

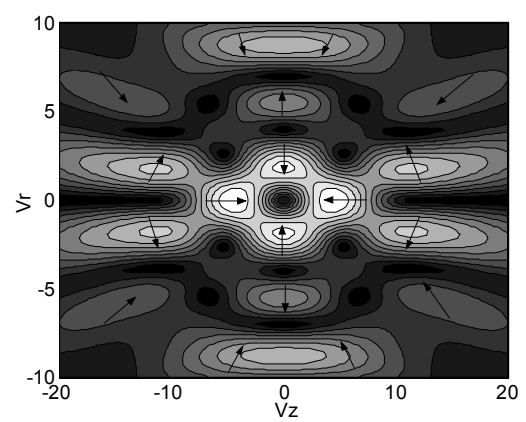

(b)

Figure 4. The (a) intensity distribution and corresponding (b) optical gradient force pattern for $\beta_{1}=2.5$ and $C=1.0$, respectively.

Arrows indicate the force direction.

appears outside of center main center intensity peak. Figure 4(b) shows that center optical trap comes into being, and simultaneously, more complicate force pattern also occur outside of this main trap. From above all optical gradient evolution process, it can be given that the beam parameter and vary rate parameter can be used to alter intensity and corresponding optical gradient force distributions in focal region of Bessel-Gauss beam remarkably.

\section{CONCLUSIONS}

The optical gradient force in the focal region of radial varying polarization Bessel-Gauss beam is investigated numerically by means of vector diffraction theory. Simulation results show that the beam parameter and vary rate parameter affect the optical gradient force pattern very considerably, and some novel force distribution patterns may come into being, which indicates that the focusing of radial varying polarization Bessel-Gauss beam can be used to construct optical traps.

\section{ACKNOWLEDGMENTS}

This work was supported by National Natural Science Foundation of China (60708002, 60878024, 60778022), China Postdoctoral Science Foundation (20080430086), Shanghai Postdoctoral Science Foundation of China (08R214141), and the Innovation Fund Project For Graduate Student of Shanghai (JWCXSL1002). 


\section{REFERENCES}

[1] Ashkin, A., Dziedzic, J.M., Bjorkholm, J.E. and Chu, S. (1986) Observation of a single-beam gradient force optical trap for dielectric particles. Optics Letters, 11, 288- 290.

[2] MacDonald, M.P., Paterson, L., Volke-Sepulveda, K., Arlt, J., Sibbett, W. and Dholakia, K. (2002) Creation and manipulation of three-dimensional optically trapped structures. Science, 296, 1101-1103.

[3] Vladlen, G.S., Anton, S.D., Andrei, V.R., Wieslaw, K. and Yuri, S.K. (2009) Optical guiding of absorbing nanoclusters in air. Optics Express, 17, 5743-5757.

[4] Grier, D.G. (2003) A revolution in optical manipulation. Nature, 424, 810-816.

[5] Wang, M.D., Schnitzer, M.J., Yin, H., Landick, R., Gelles, J. and Block, S.M. (1998) Force and velocity measured for single molecules of RNA polymerase. Science, 282, 902-907.

[6] Eriksen, R.L., Mogensen, P.C. and Glückstad, J. (2002) Multiple-beam optical tweezers generated by the generalized phase-contrast method. Optics Letters, 27, 267-269.

[7] Curtis, J.E., Koss, B.A. and Grier, D.G. (2002) Dynamic holographic optical tweezers. Optics Communications, 207, 169-175.

[8] Visscher, K. and Brakenhoff, G.J. (1992) Theoretical study of optically induced forces on spherical particles in a single beam trap I: Rayleigh scatterers, Optik, 89,
174-180.

[9] Durnin, J. (1987) Exact solutions for nondiffracting beams. I. The scalar theory. Journal of the Optical Society America Associates, 4, 651-654.

[10] Li, Y., Gurevich, V., Kirchever, M., Katz, J. and Marom, E. (2001) Propagation of anistropic Bessel-Gaussian beams: sidelobe control, mode selection, and field depth. Applied Optics, 40, 2709-2721.

[11] Yang, Y. and Li, Y. (2007) Spectral shifts and spectral switches of a pulsed Bessel-Gauss beam from a circular aperture in the far field. Optics \& Laser Technology, 39, $1478-1484$.

[12] Igor, A. and Litvin Andrew, F. (2008) Bessel-Gauss resonator with internal amplitude filter. Optics Communications, 281, 2385-2392.

[13] Zhan, Q. and Leger, J.R. (2002) Focus shaping using cylindrical vector beams. Optics Express, 10, 324-330.

[14] Youngworth, K.S. and Brown, T.G. (2000) Focusing of high numerical aperture cylindrical-vector beams. Optics Express, 7, 77-87.

[15] Elijah, Y., Yew, S., Colin, J. and Sheppard, R. (2007) Tight focusing of radially polarized Gaussian and BesselGaussian beams. Optics Letters, 32, 3417-3419.

[16] Garces-Chaves, V., McGloin, D., Melville, H., Sibbett, W. and Dholakia, K. (2002) Simultaneous micromanipulation in multiple planes using a self reconstructing light beam. Nature, 419, 145-148. 